Article

\title{
Non-Gaussianity of Four-Photon Superpositions of Fock States
}

\author{
Miguel Citeli de Freitas ${ }^{1}$ and Viktor V. Dodonov ${ }^{1,2, *(D)}$ \\ 1 Institute of Physics, University of Brasilia, P.O. Box 04455, Brasilia 70919-970, Brazil; \\ miguelciteli@hotmail.com \\ 2 International Center for Physics, University of Brasilia, Brasilia 70919-970, Brazil \\ * Correspondence: vdodonov@unb.br
}

check for

updates

Citation: De Freitas, M.C.;

Dodonov, V.V. Non-Gaussianity of Four-Photon Superpositions of Fock States. Quantum Rep. 2021, 3, 350-365. https://doi.org/10.3390/ quantum 3030022

Academic Editor: Yong Siah Teo

Received: 12 June 2021

Accepted: 28 June 2021

Published: 1 July 2021

Publisher's Note: MDPI stays neutral with regard to jurisdictional claims in published maps and institutional affiliations.

Copyright: (c) 2021 by the authors. Licensee MDPI, Basel, Switzerland. This article is an open access article distributed under the terms and conditions of the Creative Commons Attribution (CC BY) license (https:// creativecommons.org/licenses/by/ $4.0 /)$.

\begin{abstract}
We study two families of four-photon superpositions of the Fock states: even vacuum squeezed states (EVSS) and orthogonal-even coherent states (OECS). These families are distinguished due to several properties: for certain values of parameters, they give the fourth-order uncertainty products close to the known minimal value (which is lower than for the Gaussian states); they have equal dimensionless values of the second- and fouth-order moments of the coordinate and momentum for all values of parameters; they possess zero covariances for all values of parameters. Since these states are obviously non-Gaussian, we consider them as good candidates to compare several different measures of non-Gaussianity proposed by different authors for the past fifteen years. The reference Gaussian states in all examples are thermal states dependent on a single parameter (an effective temperature or the coordinate variance). We analyze the measures based on the normalized Hilbert-Schmidt distance and the relative entropy (introduced by Genoni-Paris-Banaszek), the fidelity measure (Ghiu-Marian-Marian) and its logarithmic analog (Baek-Nha), as well as the Mandilara-Karpov-Cerf "Gaussianity parameter". These measures are compared with the kurtosis of the coordinate probability density and with the non-Gaussian behavior of the Wigner function.
\end{abstract}

Keywords: four-photon superpositions; even vacuum squeezed states; orthogonal-even coherent states; kurtosis; Wigner function

\section{Introduction}

Non-Gaussian states play an important role in quantum optics. It is enough to remember that the basic Fock states are strongly non-Gaussian. Many families of such states were studied during recent decades: see, e.g., the review [1]. Recently, a new wave of interest in non-Gaussian states has grown due to possible applications in different areas of quantum information [2-6]. In this context, the problem of classifying and quantifying the degree of non-Gaussianity of quantum states has attracted the attention of many researchers in recent years [7-41].

It is well known that the following equality holds for any Gaussian state (either pure or mixed):

$$
\left\langle\hat{x}^{4}\right\rangle=3\left\langle\hat{x}^{2}\right\rangle^{2}
$$

Therefore, one may be tempted to consider the normalized kurtosis as a simple measure of non-Gaussianity

$$
\mathcal{K} \equiv\left\langle\hat{x}^{4}\right\rangle /\left(3\left\langle\hat{x}^{2}\right\rangle^{2}\right)-1,
$$

which can be easily calculated for many quantum states. However, this parameter has many disadvantages. In particular, it depends on the choice of the representation, insofar as the forms of the wave functions (or density matrices) in the coordinate and momentum representations can be quite different in the most general case.

For this reason, the main trend is to use the statistical operator $\hat{\rho}$ of the system and compare it somehow with the statistical operator $\hat{\rho}_{G}$ of some reference Gaussian state. Obviously, such measures of non-Gaussianity do not depend on the choice of representation. At this point, one can remember that all non-Gaussian states are "nonclassical". Therefore, 
it seems natural to look for possible correlations between various measures of "nonGaussianity" and measures of "non-classicality". The latter ones are frequently based on some kinds of distances between the states under study and certain states (or families of states) selected as reference ones [42-52]. The main and simplest ingredient of these approaches is the trace

$$
T \equiv \operatorname{Tr}\left(\hat{\rho} \hat{\rho}_{G}\right)=\left\langle\psi\left|\hat{\rho}_{G}\right| \psi\right\rangle,
$$

where the second equality holds for any pure state $\hat{\rho}=|\psi\rangle\langle\psi|$. Following this line of reasoning, the authors of paper [7] introduced the following measure of non-Gaussianity:

$$
\delta_{T}[\hat{\rho}]=\frac{\operatorname{Tr}\left[\left(\hat{\rho}-\hat{\rho}_{G}\right)^{2}\right]}{2 \operatorname{Tr}\left(\hat{\rho}^{2}\right)}=\frac{\operatorname{Tr}\left(\hat{\rho}^{2}+\hat{\rho}_{G}^{2}-2 \hat{\rho} \hat{\rho}_{G}\right)}{2 \operatorname{Tr}\left(\hat{\rho}^{2}\right)},
$$

where $\hat{\rho}_{G}$ is the unique Gaussian state with the same first- and second-order moments of quadrature variables as in the state $\hat{\rho}$. For pure quantum states with $\operatorname{Tr}\left(\hat{\rho}^{2}\right)=1$, the right-hand side of Equation (4) is simplified as

$$
\delta_{T}[|\psi\rangle\langle\psi|]=\frac{1}{2}\left[1+\operatorname{Tr}\left(\hat{\rho}_{G}^{2}\right)\right]-\left\langle\psi\left|\hat{\rho}_{G}\right| \psi\right\rangle .
$$

Several authors proposed simple measures containing the only positive parameter $\left\langle\psi\left|\hat{\rho}_{G}\right| \psi\right\rangle$ in the case of pure quantum states (only this case is considered in the present paper). One example is the fidelity-based measure $[17,19] \delta_{F}[\hat{\rho}]=1-\sqrt{\mathcal{F}\left(\hat{\rho}, \hat{\rho}_{G}\right)}$, where $\mathcal{F}\left(\hat{\rho}, \hat{\rho}_{G}\right)$ is the fidelity [53] between the states $\hat{\rho}$ and $\hat{\rho}_{G}$. For pure quantum states, this definition is reduced to

$$
\delta_{F}[|\psi\rangle\langle\psi|]=1-\sqrt{\left\langle\psi\left|\hat{\rho}_{G}\right| \psi\right\rangle} .
$$

The measure $\sqrt{1-\mathcal{F}\left(\hat{\rho}, \hat{\rho}_{G}\right)}$ was considered in [38]. One more measure was proposed in paper [37]:

The relative entropy

$$
\mathcal{N}_{g}[|\psi\rangle\langle\psi|]=-\ln \left(\left\langle\psi\left|\hat{\rho}_{G}\right| \psi\right\rangle\right) .
$$

$$
\delta_{E}[\hat{\rho}]=\operatorname{Tr}[\hat{\rho} \ln (\hat{\rho})]-\operatorname{Tr}\left[\hat{\rho}_{G} \ln \left(\hat{\rho}_{G}\right)\right]
$$

was suggested as a measure of non-Gaussianity in [8] (see also [19]). Similar measures were suggested, e.g., in [15] (using the Wehrl entropy in terms of the Husimi $Q$-function). For pure quantum states, we have the formula $\delta_{E}[\hat{\rho}]=-\operatorname{Tr}\left[\hat{\rho}_{G} \ln \left(\hat{\rho}_{G}\right)\right]$, which does not contain the quantum state $|\psi\rangle$ explicitly.

Our interest in the problem of non-Gaussianity originated from studies of various generalizations of uncertainty relations. It is well known that all pure Gaussian states minimize the Robertson-Schrödinger uncertainty relation $[54,55]$

$$
\mathcal{D} \equiv \sigma_{x x} \sigma_{p p}-\sigma_{x p}^{2}-\hbar^{2} / 4 \geq 0 .
$$

Several generalizations of Inequality (9) were aimed at the inclusion of higher-order statistical moments of canonical variables [56-58]. In particular, it can be interesting to know the minimal value of the product $\Pi^{(4)}=\left\langle\hat{x}^{4}\right\rangle\left\langle\hat{p}^{4}\right\rangle$. In view of identity (1), $\Pi^{(4)} \geq 9 \hbar^{4} / 16$ for any Gaussian state. However, it was shown in [56] that for specific superpositions of the vacuum and fourth excited Fock states one can achieve the value $\Pi^{(4)} \approx 0.49$. The best known lower limit of $\Pi^{(4)}$ was calculated numerically in Reference [59] for specific superpositions of the four-photon Fock states $|4 k\rangle$ with $0 \leq k \leq 6$. Since all states minimizing $\Pi^{(4)}$ are non-Gaussian, our initial idea was to try to connect properties of the four-photon superpositions with some measures of non-Gaussianity.

Looking at inequality Equation (9), one can think that the quantity $\mathcal{D}$ itself can be considered as some measure of non-Gaussianity of pure quantum states, as soon as $\mathcal{D} \equiv 0$ for all Gaussian pure states. We may call such measures (including some monotonous functions of $\mathcal{D}$ ) as $\mathcal{D}$-measures. An example of such an approach is paper [39], where 
generalizations to the case of mixed quantum states were made on the basis of the so-called Wigner-Yanase skew information [60].

Another idea is to replace zero in the right-hand side of Equation (9) with some function containing some measure of non-Gaussianity. This way was taken in papers [16,21], where the following Gaussianity parameter $g$ was introduced exactly for this purpose:

$$
g=\operatorname{Tr}\left(\hat{\rho} \hat{\rho}_{G}\right) / \operatorname{Tr}\left(\hat{\rho}_{G}^{2}\right) .
$$

This definition holds for mixed quantum states as well as for pure ones. Obviously, $g=1$ for any Gaussian state. Unfortunately, there exist non-Gaussian states with $g=1$ (an example is given in Section 4). This is a disadvantage of the definition in Equation (10) (whereas $\delta_{T}[\hat{\rho}]=0$ for Gaussian states only). However, it can be useful for detecting the entanglement [30].

Various families of quantum states were analyzed in connection with the non-Gaussianity measures described above. We give a brief and certainly incomplete list of publications mentioning pure quantum states only. Single Fock states were considered in $[7,17,22,39]$. A table comparing different measures for these states can be found in [39]. Superpositions of two Fock states were studied in $[8,9,39]$. Various superpositions of two coherent states $|\alpha\rangle$ and $|-\alpha\rangle$ with different weights and phases were considered in papers $[7,9,24,26,27,37-39]$. Such superpositions include, in particular, the so-called even/odd coherent states, introduced in [61] and frequently considered as simple models of the "Schrödinger cat states"; for numerous generalizations, one can consult, e.g., References [62,63]. Various families of photon-added and photon-subtracted states were considered in papers [14,22-24,27,29,35-37,39,64]. Many examples related to all these states were considered in [40]. Triple photon states were studied in [6].

In this paper, we add two interesting families of pure non-Gaussian states to this list. From all known measures, we choose five examples in which all ingredients can be analytically calculated rather easily: Equations (5)-(8) and (10). In addition, we study the behavior of kurtosis (Equation (2)), which shows several interesting properties. A similar analysis in the case of cat states was performed in paper [26]. Two selected families are special cases of general four-photon superpositions of the Fock states $\sum_{n=0}^{\infty} c_{n}|4 n\rangle$. There are two motivations for our choice. The first one originated from the observation that an almost absolute minimum of the product $\Pi^{(4)}$ can be observed in these states (for an appropriate choice of parameters). The second reason is due to exceptional properties of the four-photon superpositions discussed in Section 2.

The first example, considered in detail in Section 3, is the superposition of two squeezed vacuum states with opposite squeezing parameters [65]:

$$
|z\rangle_{+}=B[\hat{S}(z)+\hat{S}(-z)]|0\rangle, \quad \hat{S}(z) \equiv \exp \left[\frac{1}{2}\left(z \hat{a}^{+2}-z^{*} \hat{a}^{2}\right)\right] .
$$

Using the well-known methods of the $s u(1,1)$ algebra (see, e.g., [66,67]), the exponential in the definition of the squeezing operator $\hat{S}(z)$ can be disentangled as

$$
\hat{S}(z)=\hat{U}(z) \exp \left[-\frac{1}{2} \ln [\cosh (|z|)]\left(\hat{a} \hat{a}^{\dagger}+\hat{a}^{\dagger} \hat{a}\right)\right] \hat{U}^{\dagger}(-z),
$$

where

$$
\hat{U}(z)=\exp \left[\frac{z \tanh (|z|)}{2|z|} \hat{a}^{\dagger 2}\right]
$$

Consequently,

$$
\hat{S}(z)|0\rangle=[\cosh (|z|)]^{-1 / 2} \sum_{n=0}^{\infty} \frac{\sqrt{(2 n) !}}{2^{n}(n) !}\left[\frac{z}{|z|} \tanh (|z|)\right]^{n}|2 n\rangle,
$$


so we have the following expansion of the state Equation (11) over the Fock basis for $z=r e^{i \theta}$ with $r \geq 0$ :

$$
|z\rangle_{+}=\mathcal{N} \sum_{n=0}^{\infty} \frac{\sqrt{(4 n) !}}{2^{2 n}(2 n) !}\left[e^{i \theta} \tanh (r)\right]^{2 n}|4 n\rangle .
$$

Similar superpositions with $\theta=0$ were considered recently in [33]. More general superpositions of squeezed states were studied in $[65,68,69]$, but we confine ourselves here to the states in Equation (11). We shall call them even vacuum squeezed states (EVSS).

The second family of states, considered in Section 4, consists of superpositions of four coherent states with equal amplitudes, but with phases shifted by $\pi / 2$ :

$$
\begin{gathered}
|\psi\rangle_{4 \alpha}=B \sum_{k=0}^{3}|\alpha \exp (i k \pi / 2)\rangle=\mathcal{N} \sum_{n=0}^{\infty} \frac{\alpha^{4 n}}{\sqrt{(4 n) !}}|4 n\rangle \\
B^{2}=\frac{\exp \left(|\alpha|^{2}\right)}{16 E_{4}\left(|\alpha|^{2}\right)}, \quad \mathcal{N}^{2}=\frac{1}{E_{4}\left(|\alpha|^{2}\right)}, \quad E_{4}(x) \equiv \sum_{n=0}^{\infty} \frac{x^{4 n}}{(4 n) !}=\frac{1}{2}[\cosh (x)+\cos (x)] .
\end{gathered}
$$

These kinds of states were studied for a long time from different points of view. They were named "four-photon states" [70], "orthogonal-even coherent states" [71], "pair cat states" [72], "compass states" [73], and "four-headed cat states" [74]. Recently, such states were considered in studies [33,75,76]. Following [71], we use the abbreviation OECS for the state Equation (15).

\section{Non-Gaussianity of Arbitrary Four-Photon Superpositions and Mixtures}

The four-photon superpositions $\sum_{n=0}^{\infty} c_{n}|4 n\rangle$ possess several specific properties, which permit us to simplify many calculations. The first feature is the existence of the following simple relations between the lowest order statistical moments:

$$
\langle\hat{x}\rangle=\langle\hat{p}\rangle=\langle\hat{x} \hat{p}+\hat{p} \hat{x}\rangle=0, \quad\left\langle\hat{x}^{2}\right\rangle=\left\langle\hat{p}^{2}\right\rangle, \quad\left\langle\hat{x}^{4}\right\rangle=\left\langle\hat{p}^{4}\right\rangle .
$$

These are consequences of the relations between the quadratures and annihilation/creation operators,

$$
\hat{x}=\left(\hat{a}+\hat{a}^{\dagger}\right) / \sqrt{2}, \quad \hat{p}=\left(\hat{a}-\hat{a}^{\dagger}\right) /(i \sqrt{2}),
$$

(hereafter, we use dimensionless variables with $\hbar=1$ ). Hence, the Gaussian reference state for any four-photon superposition is described by means of the thermal statistical operator, which depends on the only parameter $\left\langle\hat{x}^{2}\right\rangle$. In particular, the corresponding Gaussian Wigner function and coordinate probability density have the following forms:

$$
W_{G}(q, p)=\left\langle\hat{x}^{2}\right\rangle^{-1} \exp \left[-\frac{q^{2}+p^{2}}{2\left\langle\hat{x}^{2}\right\rangle}\right], \quad \rho_{G}(q, q)=\left(2 \pi\left\langle\hat{x}^{2}\right\rangle\right)^{-1 / 2} \exp \left(-\frac{q^{2}}{2\left\langle\hat{x}^{2}\right\rangle}\right),
$$

Respectively,

$$
\operatorname{Tr}\left(\hat{\rho}_{G}^{2}\right)=\sigma^{-1}, \quad \sigma \equiv 2\left\langle\hat{x}^{2}\right\rangle .
$$

The normalized thermal statistical operator of the harmonic oscillator can also be written in terms of the annihilation/creation operators as

$$
\hat{\rho}_{G}=\left(1-e^{-\beta}\right) \exp \left(-\beta \hat{a}^{\dagger} \hat{a}\right),
$$

where the "inverse temperature" parameter $\beta$ can be expressed in terms of parameter $\sigma$ as follows,

$$
e^{-\beta}=\frac{\sigma-1}{\sigma+1}
$$


Consequently, the operator $\hat{\rho}_{G}$ is diagonal in the Fock basis:

$$
\hat{\rho}_{G}=\frac{2}{\sigma+1} \sum_{n=0}^{\infty}\left(\frac{\sigma-1}{\sigma+1}\right)^{n}|n\rangle\langle n| .
$$

The relative entropy measure of non-Gaussianity for pure quantum states can be written as follows,

$$
\delta_{E}[\hat{\rho}]=\frac{\beta}{e^{\beta}-1}-\ln \left(1-e^{-\beta}\right)=\frac{\sigma+1}{2} \ln (\sigma+1)-\frac{\sigma-1}{2} \ln (\sigma-1)-\ln 2 .
$$

Now, let us consider an arbitrary operator $\hat{\rho}=\sum_{m, n} \rho_{m n}|m\rangle\langle n|$ and and arbitrary diagonal operator (in the Fock basis) $\hat{\rho}_{d}=\sum_{n} p_{n}|n\rangle\langle n|$. The trace of their product contains only diagonal elements $\rho_{n n}$ :

$$
\operatorname{Tr}\left(\hat{\rho} \hat{\rho}_{d}\right) \equiv T=\sum_{n} \rho_{n n} p_{n}
$$

Formula (24) tells us that the measures (5)-(7) and (10) do not depend on the phases $\theta$ and $\phi$ of the complex numbers $z$ and $\alpha$ characterizing the states $|z\rangle_{+}$Equation (11) and $|\psi\rangle_{4 \alpha}$ Equation (15). The concrete dependences on $|z|$ and $|\alpha|$ of the cited measures are considered in the following sections. For pure quantum states considered in this paper, other non-Gaussianity measures can be written in terms of $T$ and $\sigma$ as follows:

$$
\delta_{T}=\frac{1+\sigma}{2 \sigma}-T, \quad \delta_{F}=\sqrt{1-T}, \quad \mathcal{N}_{g}=-\ln (T), \quad g=\sigma T .
$$

\section{Non-Gaussianity Measures in EVSS}

In view of Formula (13), the wave function of the squeezed state in the coordinate representation $\psi_{z}(x) \equiv\langle x|\hat{S}(z)| 0\rangle$ can be written as a series over the Hermite polynomials:

$$
\psi_{z}(x)=\frac{\exp \left(-x^{2} / 2\right)}{[\sqrt{\pi} \cosh (|z|)]^{1 / 2}} \sum_{n=0}^{\infty}\left(\frac{z \tanh (|z|)}{4|z|}\right)^{n} \frac{H_{2 n}(x)}{n !} .
$$

Using formula 5.12.1.4 from [77],

$$
\sum_{k=0}^{\infty} \frac{t^{k}}{k !} H_{2 k}(x)=(1+4 t)^{-1 / 2} \exp \left(\frac{4 t x^{2}}{1+4 t}\right),
$$

one can obtain the following expression:

$$
\psi_{z}(x)=\pi^{-1 / 4}\left[u_{+}(z)\right]^{-1 / 2} \exp \left[-\frac{u_{-}(z)}{2 u_{+}(z)} x^{2}\right]
$$

where

$$
u_{ \pm}(z)=\cosh (|z|) \pm \frac{z}{|z|} \sinh (|z|)=u_{\mp}(-z) .
$$

Consequently, the wave function $\Psi_{z}(x)$ of EVSS Equation (11) in the coordinate representation has the form

$$
\Psi_{z}(x)=N\left\{u_{+}^{-1 / 2} \exp \left[-\frac{u_{-} x^{2}}{2 u_{+}}\right]+u_{-}^{-1 / 2} \exp \left[-\frac{u_{+} x^{2}}{2 u_{-}}\right]\right\},
$$

where $N$ is the normalization factor. The following relations are useful for calculations:

$$
\begin{gathered}
u_{+} u_{-}^{*}=1+i \kappa, \quad \kappa=\sinh (2 r) \sin (\theta) \\
\left|u_{ \pm}\right|^{2}=\cosh (2|z|) \pm \sinh (2|z|) \cos (\theta) \\
\left|u_{+}\right|^{2}\left|u_{-}\right|^{2}=1+\kappa^{2}, \quad\left|u_{+}\right|^{2}+\left|u_{-}\right|^{2}=2 \cosh (2|z|) .
\end{gathered}
$$


The probability density has the form

$$
\begin{aligned}
& \left|\Psi_{z}(x)\right|^{2}=|N|^{2}\left\{\frac{1}{\left|u_{+}\right|} \exp \left(-\frac{x^{2}}{\left|u_{+}\right|^{2}}\right)+\frac{1}{\left|u_{-}\right|} \exp \left(-\frac{x^{2}}{\left|u_{-}\right|^{2}}\right)\right. \\
& \left.+2 \operatorname{Re}\left[\left(u_{+} u_{-}^{*}\right)^{-1 / 2} \exp \left(-\frac{x^{2} \cosh (2|z|)}{u_{+} u_{-}^{*}}\right)\right]\right\} .
\end{aligned}
$$

After the integration, using identities Equations (31)-(33), we arrive at the remarkable result that the normalization constant depends on the only parameter $c \equiv \sqrt{\cosh (2|z|)}$ :

$$
|N|^{2}=\frac{B}{2 \sqrt{\pi}}, \quad B=\frac{c}{1+c} .
$$

Calculating the Fourier transform of function $\psi_{z}(x)$, one obtains the wave function of the squeezed state in the momentum representation,

$$
\tilde{\psi}_{z}(p)=\pi^{-1 / 4}\left[u_{-}(z)\right]^{-1 / 2} \exp \left[-\frac{u_{+}(z)}{2 u_{-}(z)} p^{2}\right] .
$$

Consequently, $\tilde{\Psi}_{z}(p)$ is given by the same Formula (30), with $x$ replaced by $p$. Therefore, all statistical moments of the coordinate and momentum coincide in the EVSS.

Simple calculations show that the second-order moment also depends on the only parameter $c$ :

$$
\left\langle\hat{x}^{2}\right\rangle=\frac{c^{5}+1}{2 c^{2}(1+c)} \equiv \sigma / 2 .
$$

Note that $\sigma \approx c^{2}$ when $c \gg 1$. If $r \ll 1$, then, $c \approx 1+r^{2}-r^{4} / 6$ and $\sigma \approx 1+3 r^{4}$.

The fourth-order moment depends on $r$ and $\theta$ :

$$
\left\langle\hat{x}^{4}\right\rangle(r, \theta)=\frac{3 B}{4}\left\{\cosh ^{2}(2 r)+\cos ^{2}(\theta) \sinh ^{2}(2 r)+\frac{1-\sin ^{2}(\theta) \sinh ^{2}(2 r)}{[\cosh (2 r)]^{5 / 2}}\right\} .
$$

The minimum of $\left\langle\hat{x}^{4}\right\rangle(r, \theta)$ for the fixed value of $r$ is achieved for $\theta=\theta_{*}= \pm \pi / 2$, so that

$$
\left\langle\hat{x}^{4}\right\rangle\left(r, \theta_{*}\right)=\frac{3 B}{4}\left\{\cosh ^{2}(2 r)+\frac{1-\sinh ^{2}(2 r)}{[\cosh (2 r)]^{5 / 2}}\right\} .
$$

Numerical calculations yield $\left\langle\hat{x}^{4}\right\rangle_{\min }=0.6987$ and $\Pi_{\text {min }}^{(4)}=0.4882$ for $r=r_{*}=0.2690$ and $c_{*}=1.072$. This value is very close to the minimal value $\Pi_{\min }^{(4)}=0.4878$ found in study [59]. In our case, $\left\langle\hat{x}^{2}\right\rangle\left(r_{*}, \theta_{*}\right)=0.5072$, only a slightly higher value than in the vacuum state. Note that $\Pi^{(4)}\left(z_{*}\right)=7.3743\left[\Pi^{(2)}\left(z_{*}\right)\right]^{2}$. If the state were Gaussian, then the coefficient in the right-hand side would be equal to 9 .

For small values of $r$, we have $\left\langle\hat{x}^{4}\right\rangle(r, \theta) \approx(3 / 4)\left[1+2 r^{2} \cos (2 \theta)\right]$. Figure 1 shows the probability density Equation (34) for different values of the squeezing parameter $r$ and phase $\theta$. We see that the probability density looks close to the Gaussian for $r<0.5$, whereas it becomes obviously non-Gaussian for higher values of $r$. Pay attention to the inverse order of lines in the left-hand and right-hand sides of Figure 1. These parts look totally different for large values of $r$. Therefore, it is interesting to understand how the non-Gaussianity measures correlate with these visual observations. 

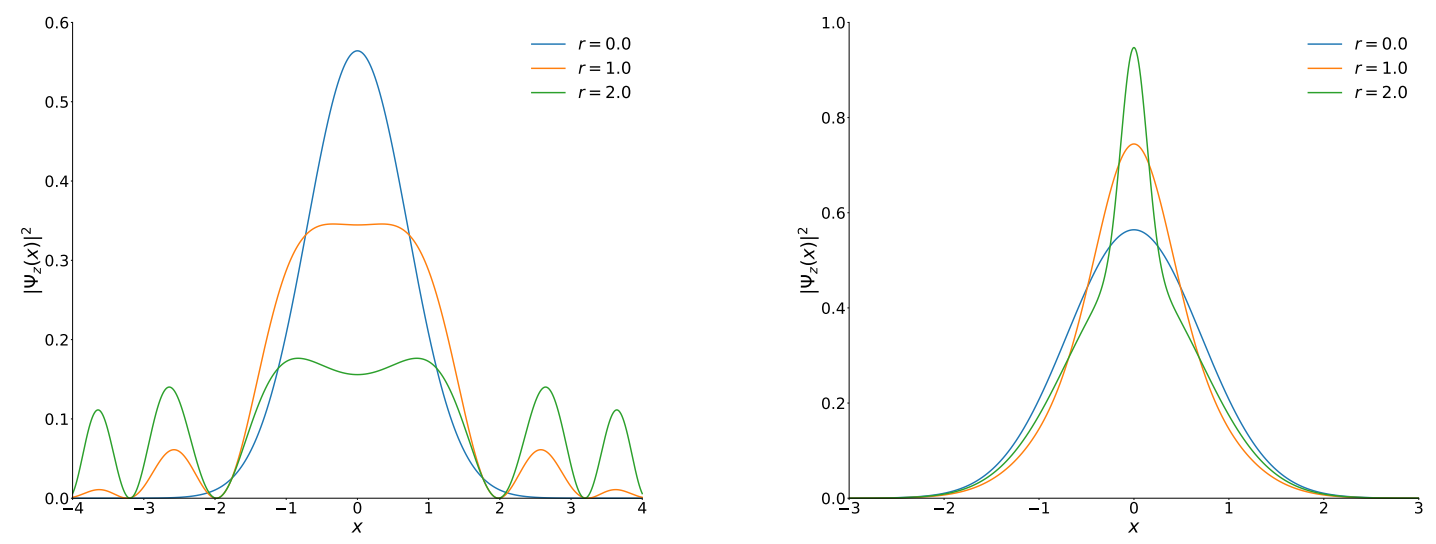

Figure 1. The probability density Equation (34) of EVSS for different values of the squeezing parameter $r$. Left: $\theta=\pi / 2$. Right: $\theta=0$.

The Wigner function of state Equation (30) equals

$$
\begin{aligned}
& W_{z}(q, p)=B\left\{\exp \left[-p^{2}\left|u_{+}\right|^{2}-q^{2}\left|u_{-}\right|^{2}+2 \kappa p q\right]+\exp \left[-p^{2}\left|u_{-}\right|^{2}-q^{2}\left|u_{+}\right|^{2}-2 \kappa p q\right]\right. \\
& \left.+2[\cosh (2 r)]^{-1 / 2} \operatorname{Re}\left(\exp \left[2 i p q \tanh (2 r) \cos (\theta)-p^{2} \frac{1-i \kappa}{\cosh (2 r)}-q^{2} \frac{1+i \kappa}{\cosh (2 r)}\right]\right)\right\} .
\end{aligned}
$$

This function is invariant with respect to the rotation by 90 degrees in the phase plane: $q \rightarrow p, p \rightarrow-q$. Note that $W_{z}(0,0)=2$ for any value of parameter $z$, as it must be for any symmetrical pure state [78]. One can verify the following expansion of Formula (40) for small values of $q$ and $p$ :

$$
W_{z}(q, p) \approx 2\left[1-2\left\langle\hat{x}^{2}\right\rangle\left(q^{2}+p^{2}\right)\right]
$$

Remember that we use dimensionless variables, assuming formally $\hbar=1$. The expansion Equation (41) indicates that function $W_{z}$ is strongly non-Gaussian, unless the squeezing parameter $r$ is close to zero. Indeed, a similar expansion of function (18) has the form

$$
W_{G}(q, p) \approx\left\langle\hat{x}^{2}\right\rangle^{-1}\left[1-\frac{q^{2}+p^{2}}{2\left\langle\hat{x}^{2}\right\rangle}\right] \text {. }
$$

Therefore, the non-Gaussianity of $W_{z}$ is clearly demonstrated by the narrow peak nearby the origin of the width proportional to $\left\langle\hat{x}^{2}\right\rangle^{-1 / 2}$ : see Figure 2.

Note that the section $W_{z}(q, 0)$ of the total Wigner function with $\theta=0$ is positive in the whole interval $-\infty<q<\infty$. However, $W_{z}(q, 0)$ with $\theta=\pi / 2$ shows strong oscillations and negative values if $r$ is not too small, in accordance with the Hudson theorem [79]. Plots of the "diagonal" section $W_{z}(q, q)$ are similar to those for $W_{z}(q, 0)$, although with an "inversion" of the left and right plots with respect to the phase $\theta$.

The sum Equation (24) with coefficients $\rho_{n n}$ following from Formula (13) seems too complicated. In this case, a simpler way to calculate the trace $\operatorname{Tr}\left(\hat{\rho} \hat{\rho}_{G}\right)$ is to use the formula $\operatorname{Tr}\left(\hat{\rho} \hat{\rho}_{G}\right)=\int W W_{G} d q d p /(2 \pi)$, which contains only Gaussian integrals. Using identity Equations (31)-(33), we obtain the formula

$$
\operatorname{Tr}\left(\hat{\rho}_{z} \hat{\rho}_{G}\right) \equiv T=\frac{2 c}{1+c}\left\{\left[\sigma^{2}+2 c^{2} \sigma+1\right]^{-1 / 2}+\left[\left(\sigma^{2}+1\right) c^{2}+2 \sigma\right]^{-1 / 2}\right\},
$$

where $\sigma=\sigma(c)$ was defined in Equation (37). As was explained in Section 2, this trace does not contain the phase $\theta$. If $\sigma \approx c^{2} \gg 1$, then $T \approx 2 /(\sigma \sqrt{3})$. 

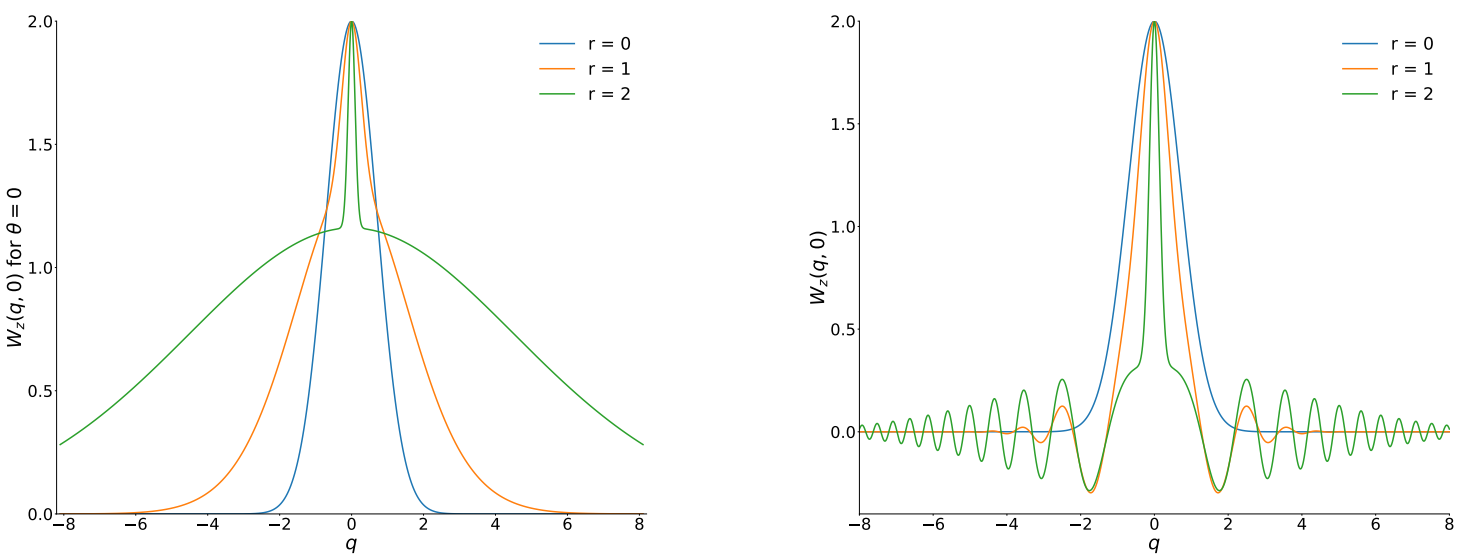

Figure 2. The section $W_{z}(q, 0)$ of EVSS for different values of the squeezing parameter $r$ and phase $\theta$. Left: $\theta=0$; $r=0,1.0,2.0$. Right: $\theta=\pi / 2$, the same values of $r$.

Plots of measures Equations (23) and (25) as functions of parameter $c$ are given in Figure 3. They are compared with the plots of the normalized kurtosis $\mathcal{K}$ (Equation (2)). Three coefficients $\delta\left[\rho_{z}\right](c)$ grow monotonously from 0 at $c=1$ to their asymptotic values as $c \rightarrow \infty$, and nothing exceptional is noticed for $c=c_{*}$. The coefficient $g(c)$ grows initially, attains a wide maximum $g_{\max } \approx 1.27$ at $r_{\max } \approx 1.33$ (or $c_{\max } \approx 2.68$ ), and decays monotonously to $g_{\infty}=2 / \sqrt{3} \approx 1.155$ as $c \rightarrow \infty$. However, the maximum of $g(c)$ apparently has no correlation with the minimum of $\left\langle\hat{x}^{4}\right\rangle$. For $r \ll 1$ we obtain $g \approx 1+9 r^{4} / 8$ and $\delta_{T}\left[\rho_{z}\right] \approx 3 r^{4} / 8$.
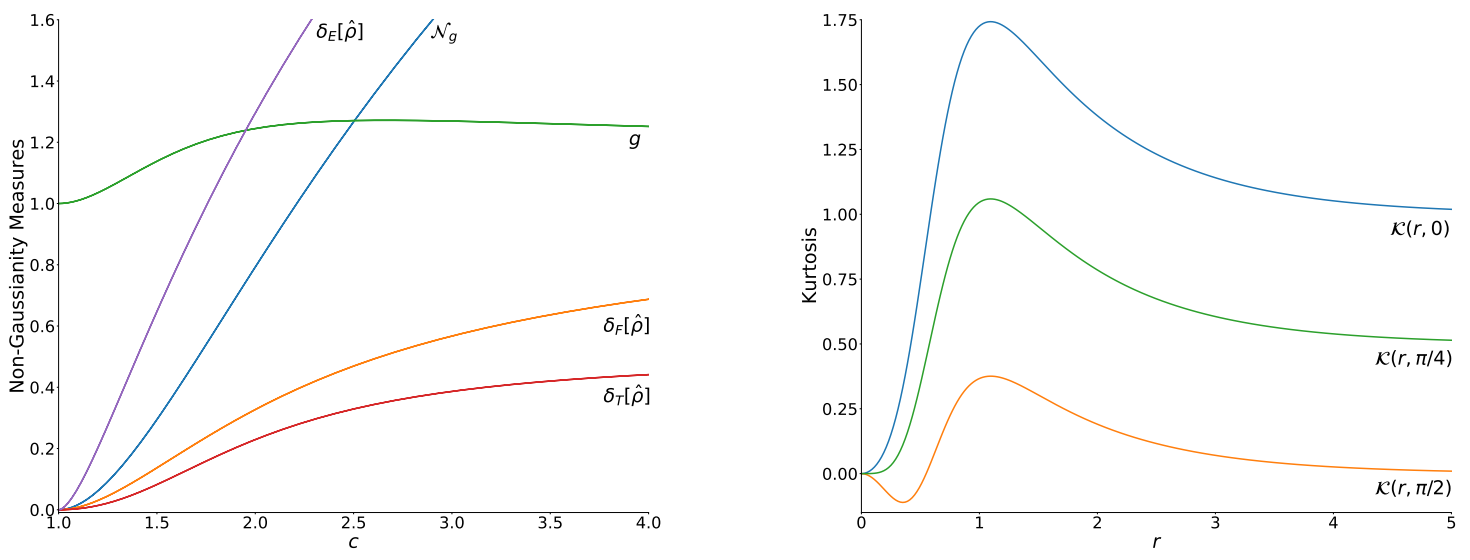

Figure 3. The behavior of the non-Gaussianity measures (left) and kurtosis (right) as functions of parameters $c$ and $r$, respectively, for the Even Vacuum Squeezed States.

The behavior of the normalized kurtosis is more interesting. For $\theta=\pi / 2, \mathcal{K}$ is negative for small values of the squeezing parameter $r$, attaining the minimal value $\mathcal{K}_{\text {min }}=-0.1111$ at $r_{\min }=0.3504$. However, $\mathcal{K}$ becomes positive for $r>0.549$, and it goes to zero as $c^{-1}$ when $c \rightarrow \infty$. On the other hand, $\mathcal{K}$ is always positive for $\theta \leq \pi / 4$. Its asymptotic behavior for $c \rightarrow \infty$ is given by the formulas $\mathcal{K} \approx 1+2 / c$ for $\theta=0$ and $\mathcal{K} \approx 1 / 2+3 /(2 c)$ for $\theta=\pi / 4$. In all cases, $\mathcal{K}$ has maximums: $\mathcal{K}_{\text {max }} \approx 0.3757$ at $r=1.1011$ if $\theta=\pi / 2, \mathcal{K}_{\max } \approx 1.0592$ at $r=1.0911$ if $\theta=\pi / 4$, and and $\mathcal{K}_{\max } \approx 1.7427$ at $r=1.0911$ if $\theta=0$. However, we do not see any correlation of these maximums with the behavior of the Wigner functions or the functions $g(c)$ and $\delta\left[\rho_{z}\right](c)$. 


\section{Non-Gaussianity Measures in OECS}

In the case of OECS Equation (15), the second-order mean value of the coordinate and momentum does not depend on the phase of complex parameter $\alpha$ [58]):

$$
\left\langle\hat{x}^{2}\right\rangle=\left\langle\hat{p}^{2}\right\rangle \equiv \sigma / 2=\frac{1}{2}+a \frac{\sinh (a)-\sin (a)}{\cosh (a)+\cos (a)}, \quad \alpha \equiv \sqrt{a} e^{i \phi} .
$$

On the other hand,

$$
\left\langle\hat{x}^{4}\right\rangle=\left\langle\hat{p}^{4}\right\rangle=\frac{3}{4}+\frac{a^{2}}{2} \cos (4 \phi)+3 \frac{a[\sinh (a)-\sin (a)]+a^{2}[\cosh (a)-\cos (a)] / 2}{\cosh (a)+\cos (a)} .
$$

The minimum $\left\langle\hat{x}^{4}\right\rangle_{\min }=0.6999$ is achieved for $\cos (4 \phi)=-1$ at $a_{*}=0.4489$.

The normalized wave function of OECS in the coordinate representation has the following form:

$$
\psi_{4 \alpha}(x)=\frac{\exp \left(-x^{2} / 2\right)}{2 \pi^{1 / 4} \sqrt{E_{4}\left(|\alpha|^{2}\right)}}\left[\cosh (\sqrt{2} \alpha x) \exp \left(-\alpha^{2} / 2\right)+\cos (\sqrt{2} \alpha x) \exp \left(\alpha^{2} / 2\right)\right] .
$$

The behavior of this function strongly depends on the phase $\phi$ of complex number $\alpha=|\alpha| e^{i \phi}$, especially if $|\alpha| \gg 1$. For example, if $|\alpha x| \ll 1$, then,

$$
\psi_{4 \alpha}(x) \approx \pi^{-1 / 4}\left[E_{4}(a)\right]^{-1 / 2}\left[\cosh \left(\alpha^{2} / 2\right)\left(1-x^{2} / 2\right)-x^{2} \alpha^{2} \sinh \left(\alpha^{2} / 2\right)\right] .
$$

If $\phi=0$ and $|\alpha| \gg 1$, we have the maximum of $\psi_{4 \alpha}(x)$ at $x=0$. The height of the maximum $\psi_{4 \alpha}(0)$ practically does not depend on $|\alpha|$. However, the width of this maximum $\delta x \sim|\alpha|^{-1}$ is strongly reduced, compared with the case of $\alpha=0$. On the other hand, for $\phi=\pi / 4$ and $|\alpha|^{2}=\pi$ we have $\psi_{4 \alpha}(0)=0$. Plots of Equation (45) with $\phi=0$ and $\phi=\pi / 4$ (when this function is real) are shown in Figure 4 for different (not very large) values of $|\alpha|$. Figure 5 shows the coordinate probability density for larger values of parameter $a=|\alpha|^{2}$. A significant difference between the cases of phases $\phi=0$ and $\phi=\pi / 4$ is clearly seen.
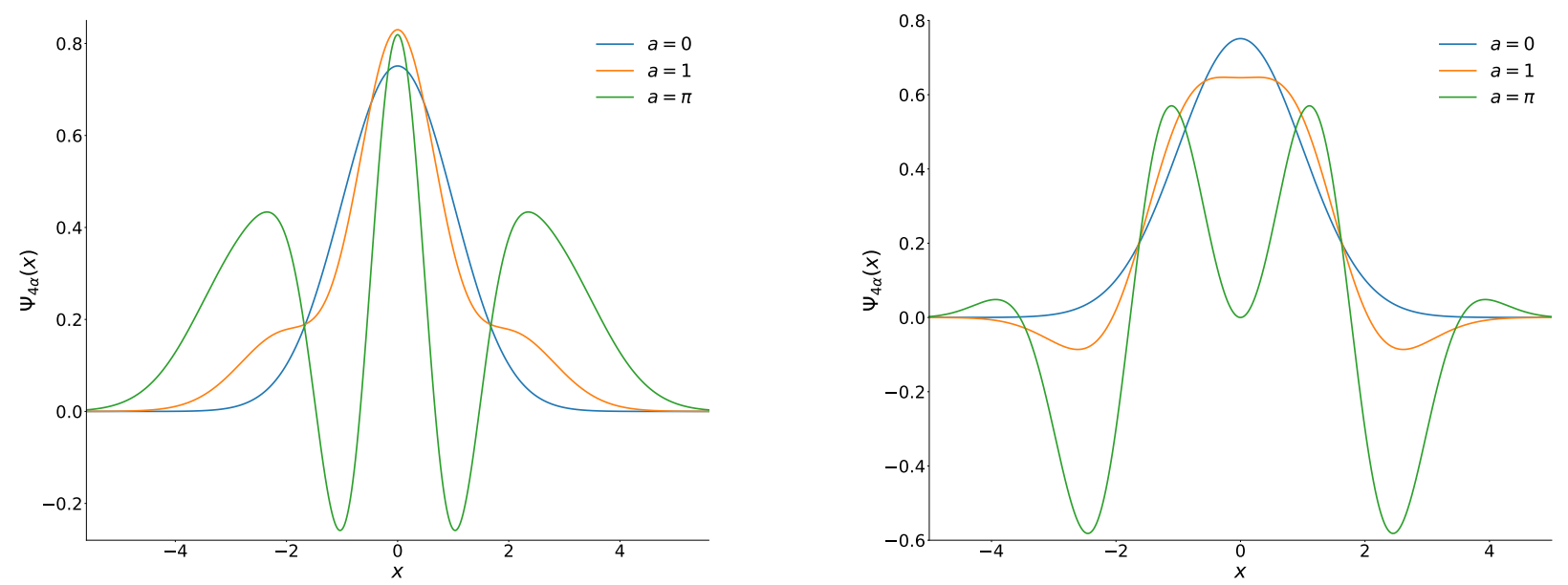

Figure 4. Real wave functions of OECS for different values of parameter $\alpha=\sqrt{a} \exp (i \phi)$. Left: $\phi=0$. Right: $\phi=\pi / 4$. 

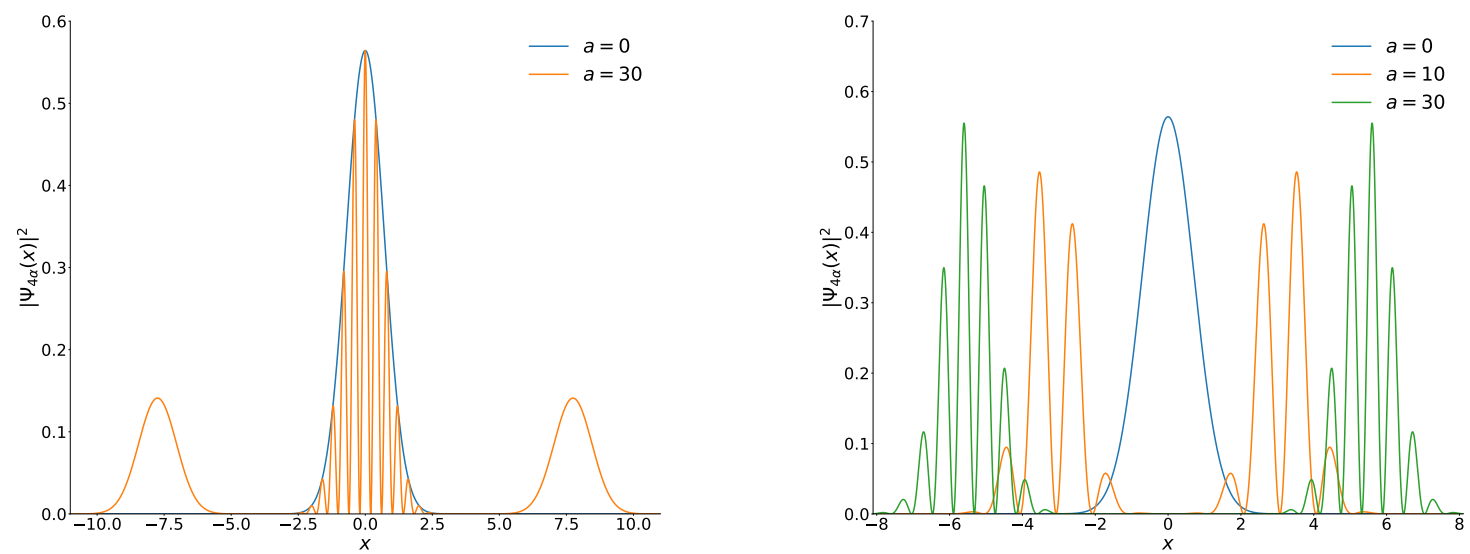

Figure 5. The coordinate probability density of OECS for different values of parameter $a=|\alpha|^{2}$ and phase $\phi$. Left: $\phi=0$. Right: $\phi=\pi / 4$.

The Wigner function is the sum of 16 exponential terms with $\alpha_{k}=\alpha i^{k}$ and $\alpha_{j}=\alpha i^{j}$,

$$
W_{4 \alpha}(q, p)=\frac{\exp \left(-q^{2}-p^{2}\right)}{8 E_{4}\left(|\alpha|^{2}\right)} \sum_{k, j=0}^{3} \exp \left[\sqrt{2} q\left(\alpha_{k}+\alpha_{j}^{*}\right)-i \sqrt{2} p\left(\alpha_{k}-\alpha_{j}^{*}\right)-\alpha_{k} \alpha_{j}^{*}\right] \text {. }
$$

Plots of the sections $W_{4 \alpha}(q, 0)$ and $\left.W_{4 \alpha}(q, q)\right)$ are given in Figure 6 for $\phi=0$ and $\alpha^{2}=0, \pi / 2, \pi$. Although the section $W_{4 \alpha}(q, 0)$ is positive in the whole interval $-\infty<q<\infty$, the section $W_{4 \alpha}(q, q)$ shows strong oscillations and negative values. In all the cases, the width of the central peak diminishes with the increase in parameter $a$. Plots of the same sections with $\phi=\pi / 4$ are given in Figure 7 for $|\alpha|=0$ and $|\alpha|=3$.
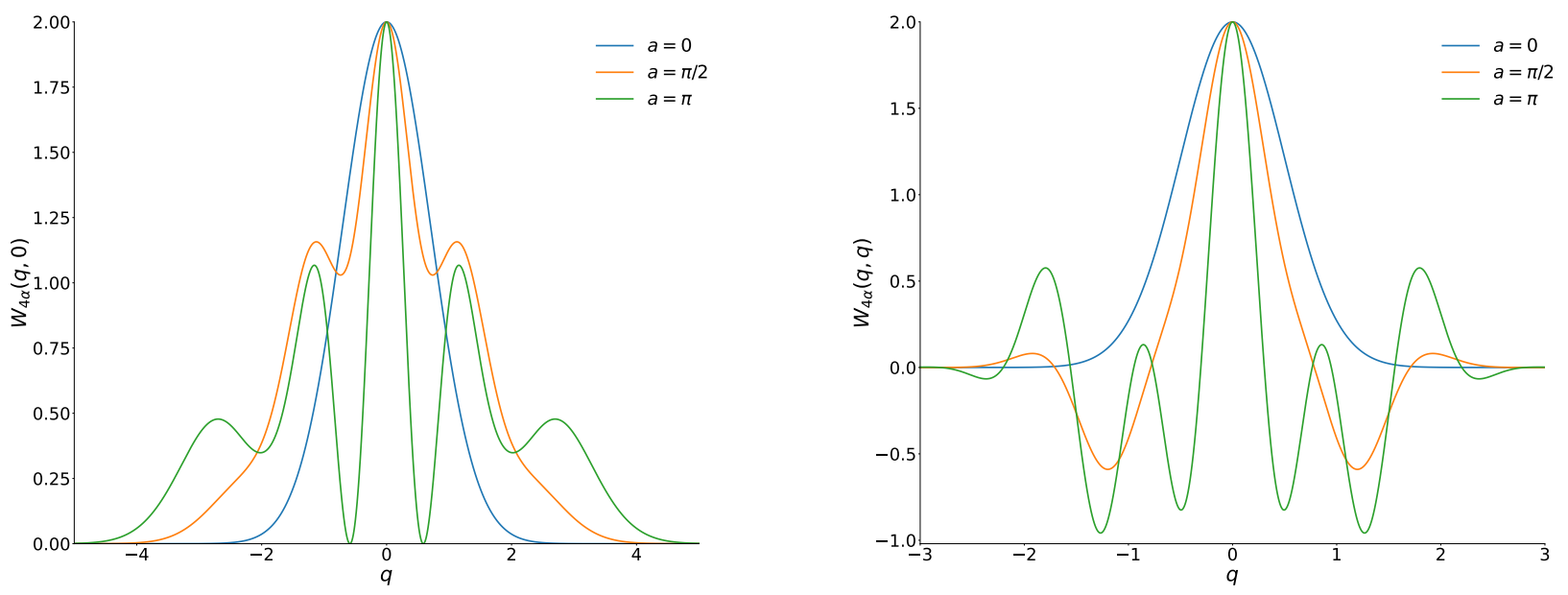

Figure 6. The sections of function $W_{4 \alpha}(q, p)$ for different values of real parameter $\alpha=\sqrt{a}$. Left: $W_{4 \alpha}(q, 0)$. Right: $W_{4 \alpha}(q, q)$. 

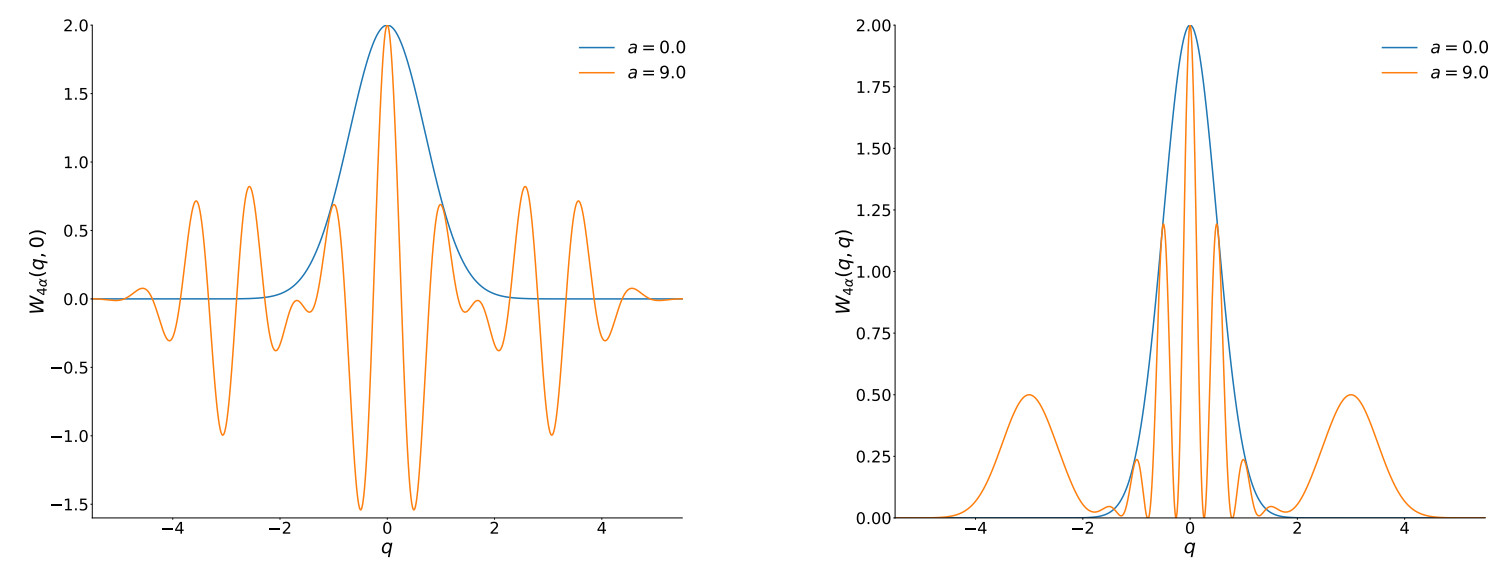

Figure 7. The sections of function $W_{4 \alpha}(q, p)$ for $\alpha=|\alpha| \exp (i \pi / 4)$. Left: $W_{4 \alpha}(q, 0)$. Right: $W_{4 \alpha}(q, q)$.

In the case involved, the trace $\operatorname{Tr}\left(\hat{\rho} \hat{\rho}_{G}\right)$ can be easily calculated by means of formula (24) because it is reduced to the simple series $E_{4}(x)$ defined in Equation (16):

$$
\operatorname{Tr}\left(\hat{\rho} \hat{\rho}_{G}\right) \equiv T=\frac{2 E_{4}\left(a e^{-\beta}\right)}{(\sigma+1) E_{4}(a)}, \quad e^{-\beta}=\frac{\sigma-1}{\sigma+1} .
$$

Plots of measures Equations (23) and (25) as functions of parameter $a$ are given in Figure 8, together with the plots of kurtosis. We see that the behavior of functions $g(a)$ and $\mathcal{K}(a)$ is quite different from the behavior of functions $g(c)$ and $\mathcal{K}(c)$ in the case of EVSS, shown in Figure 3. Function $g(a)$ is especially interesting. Indeed, function $E_{4}(x)$ behaves as $e^{x} / 4$ for $x \gg 1$. If $a \gg 1$, then $\sigma \approx 1+2 a$. In this limit case, we have $e^{-\beta} \approx a /(1+a) \approx 1-1 / a$. Neglecting the term $1 / a$, one could think that $E_{4}\left(a e^{-\beta}\right) \approx E_{4}(a)$ for $a \rightarrow \infty$, arriving at the asymptotic value $g(\infty)=2$. However, a more correct calculation yields the ratio $E_{4}\left(a e^{-\beta}\right) / E_{4}(a) \approx E_{4}(a-1) / E_{4}(a) \approx 1 / e$ for $a \gg 1$. Therefore, the correct asymptotic value is $g(\infty)=2 / e \approx 0.74$, in full accordance with Figure 8. Hence, $T \approx 2 /(e \sigma)$ when $a \gg 1$ and $\sigma \approx 1+2 a \gg 1$. The maximum value $g_{\max } \approx 1.1735$ is attained at $a=1.4615$. In addition, $g(a)<1$ for $a>2.209$.
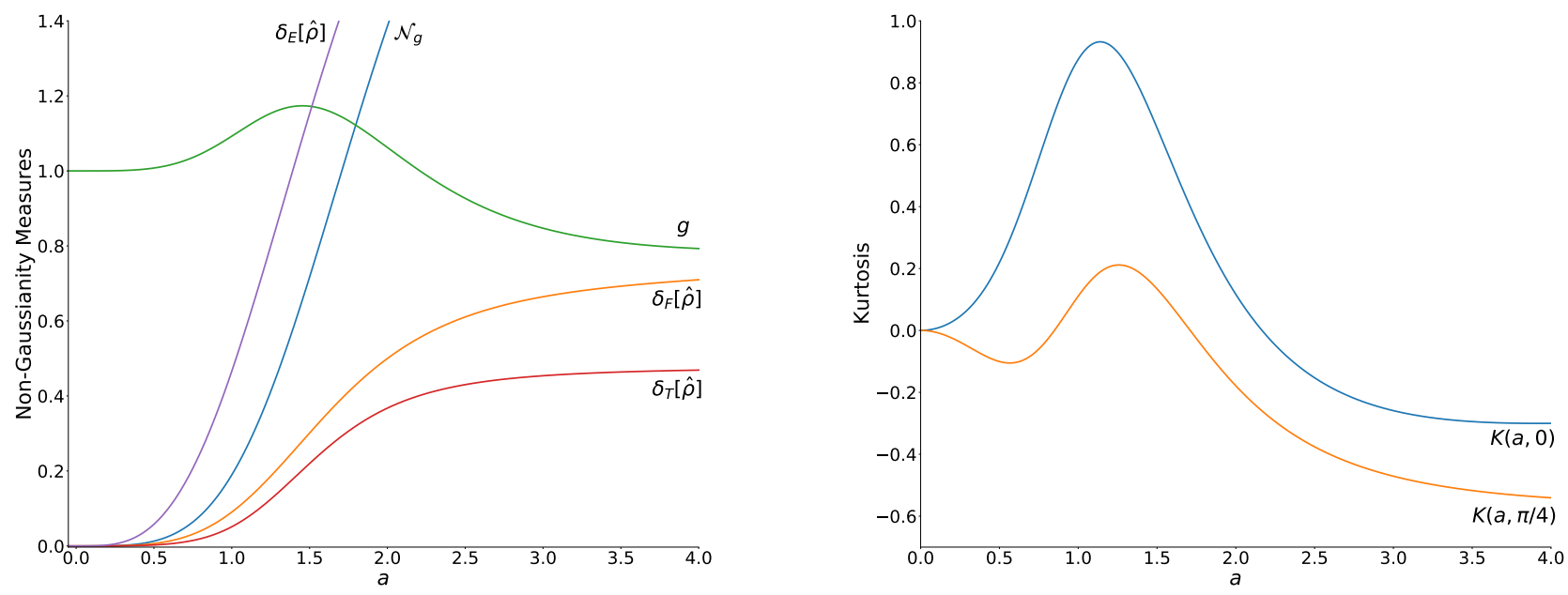

Figure 8. The behavior of the non-Gaussianity measures (left) and kurtosis (right) as functions of parameter $a$ for the Orthogonal Even Coherent States. 
The evolution of the kurtosis depends on the phase $\phi$. If $\phi=\pi / 4$, the local minimum $\mathcal{K}_{\text {min }}=-0.1052$ is achieved at $a=0.5658$ and maximum $\mathcal{K}_{\text {max }}=0.2115$ at $a=1.2577$. In addition, $\mathcal{K}=0$ at $a=0.8490$ and $a=1.7103$. If $\phi=0$, one can see the maximum $\mathcal{K}_{\text {max }}=0.9323$ at $a=1.1391$. When $a \rightarrow \infty$, the kurtosis tends to the asymptotic value $\mathcal{K}(\infty)=[\cos (4 \phi)-3] / 6$. The minimal possible asymptotic value $-2 / 3$ corresponds to $\phi=\pi / 4$. This is an example of distribution possessing the property $\left\langle\hat{x}^{4}\right\rangle \approx\left\langle\hat{x}^{2}\right\rangle^{2}$. This equality is explained by the right-hand side of Figure 5: when $a \rightarrow \infty$, the distribution (strongly non-Gaussian) is concentrated symmetrically in two small regions. On the other hand, if $\phi=0$, then the distribution remains concentrated nearby the origin, with two symmetric relatively small remote wings: see the left-hand part of Figure 5. As a consequence, the ratio $\left\langle\hat{x}^{4}\right\rangle /\left\langle\hat{x}^{2}\right\rangle^{2}$ turns out twice as large as for $\phi=\pi / 4$.

\section{Comparison of Non-Gaussianity of EVSS and OECS}

It is interesting to compare the degrees of non-Gaussianity of two families of states: EVSS and OECS. For this purpose, these degrees must be calculated for equal values of some parameter. A relevant physical parameter could be the state energy, or, equivalently for the four-photon superpositions, the coordinate variance $\sigma / 2$. However, the dependences $\sigma(r)$ (for EVSS) and $\sigma(a)$ (for OECS) are rather complicated, so that the inverse functions $r(\sigma)$ and $a(\sigma)$ can be found only numerically. For this reason, we choose the simple common parameter in the form of the inverse fidelity between each component of the superpositions and the vacuum state: $\tau=|\langle 0 \mid \alpha\rangle|^{-2}$ and $\tau=|\langle 0 \mid z\rangle|^{-2}$. Then,

$$
a=\ln (\tau), \quad \cosh (r)=\tau, \quad c=\sqrt{2 \tau^{2}-1} .
$$

Figure 9 shows the measures $\delta_{T}[\hat{\rho}](\tau)$ and $g(\tau)$ for EVSS and OECS. According to this figure, the EVSS are "more non-Gaussian" than OECS for all values of parameter $\tau$, especially for small values of this parameter. However, it is difficult to confirm this evaluation by looking at plots of the probability distributions and Wigner functions. Attempts to quantify different families of states according to their "non-Gaussianity" probably have no real sense.
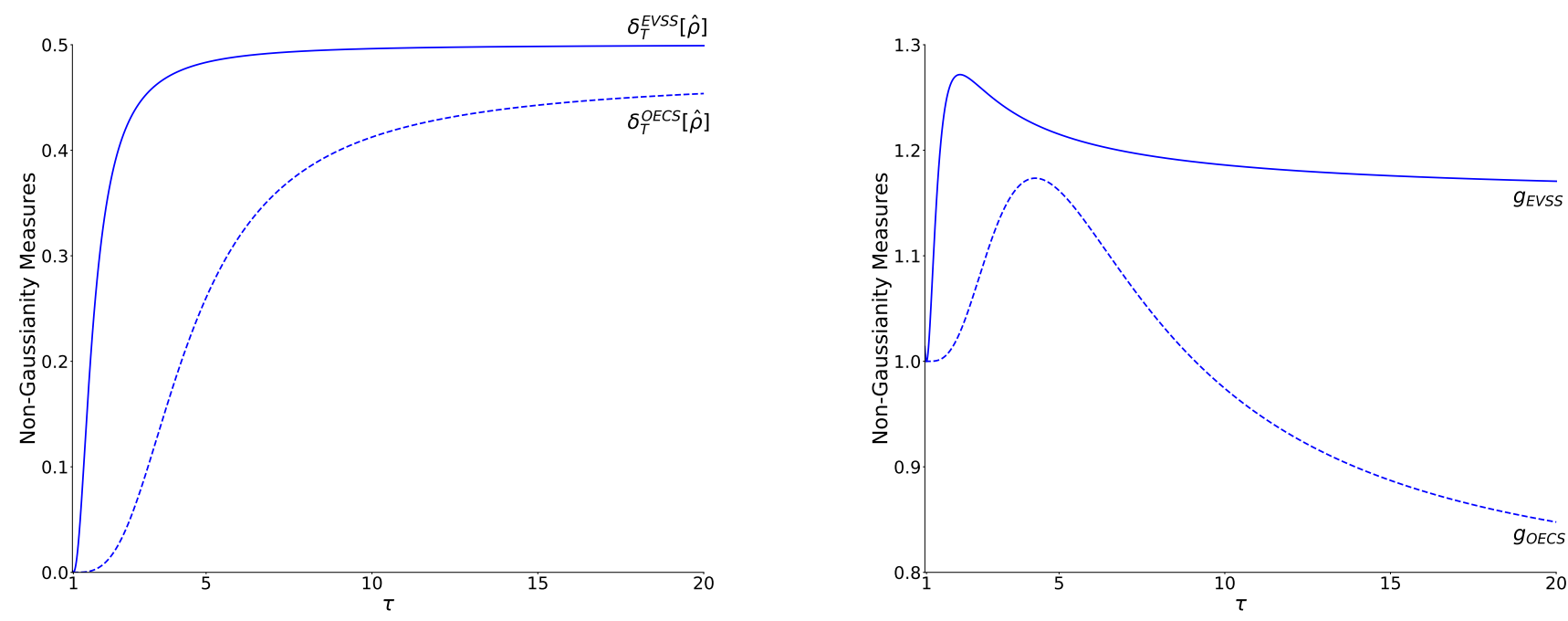

Figure 9. Non-Gaussianity measures as functions of the inverse fidelity $\tau$ between the vacuum state and components of two kinds of superpositions. (Left) The measures $\delta_{T}[\hat{\rho}](\tau)$ for EVSS and OECS; (Right) The measures $g(\tau)$ for EVSS and OECS.

\section{Conclusions}

We have compared five different measures of non-Gaussianity for two interesting four-photon superpositions of the Fock states: even vacuum squeezed states (EVSS) and orthogonal-even coherent states (OECS). Four measures show a monotonous growth when 
parameters characterizing the "size" of superpositions are increased. However, it is difficult to choose any of these measures as the "best" one: all of them seem more or less equivalent, at least for the pure quantum states studied in this paper. The behavior of the "Gaussianity" measure Equation (10) is quite different. It is non-monotonous (apparently due to the presence of the factor $\operatorname{Tr}\left(\hat{\rho}_{G}^{2}\right)$ in the denominator), so this measure can hardly be used to compare the degree of Gaussianity of different states. Moreover, all the measures do not take into account a "fine structure" of quantum states. In particular, all of them do not distinguish between the states with different phases but equal absolute values of complex parameters $z$ or $\alpha$, while the plots of the probability densities and Wigner functions show the importance of these phases. In this connection, the behavior of kurtosis (which strongly depends on the phases) can provide an important additional information. It is interesting that "large" even superpositions of coherent states (with $a \gg 1$ ) show the "sub-Gaussian" behavior. Moreover, by selecting the specific phase of parameter $\alpha$, one can obtain a ratio $\left\langle\hat{x}^{4}\right\rangle /\left(\left\langle\hat{x}^{2}\right\rangle\right)^{2}$ as close to unity as desired. On the contrary, almost all even superpositions of the vacuum squeezed states are "super-Gaussian", except for small values of complex parameter $z$ with properly chosen phases. Another example of super-Gaussian statistics was demonstrated in the recent paper [6] for the "triple photon states". The EVSS can be produced via nonlinear processes in the presence of the Kerr nonlinearity [65]. General schemes of constructing multiphoton coherent and squeezed states of arbitrary orders were given in [80-83]. Experimental schemes of detecting various non-Gaussianity measures (including the fidelity) were presented in papers $[10,13,25,41,84]$.

Author Contributions: M.C.d.F.: analytical and numerical calculations, plotting figures; V.V.D.: conceptualization, methodology, analytical calculations, and writing the paper. All authors have read and agreed to the published version of the manuscript.

Funding: This research received no external funding.

Acknowledgments: The authors thank A.C. Pedroza and A.E. Santana for the interest in the work and useful remarks. V.V.D. acknowledges the partial support of the Brazilian funding agency Conselho Nacional de Desenvolvimento Científico e Tecnológico (CNPq).

Conflicts of Interest: The authors declare no conflict of interest.

\section{References}

1. Dodonov, V.V. 'Nonclassical' states in quantum optics: A 'squeezed' review of the first 75 years. J. Opt. B Quantum Semiclass. Opt. 2002, 4, R1-R33. [CrossRef]

2. Dell'Anno, F.; De Siena, S.; Adesso, G.; Illuminati, F. Teleportation of squeezing: Optimization using non-Gaussian resources. Phys. Rev. A 2010, 82, 062329. [CrossRef]

3. Zhuang, Q.; Shor, P.W.; Shapiro, J.H. Resource theory of non-Gaussian operations. Phys. Rev. A 2018, 97, 052317. [CrossRef]

4. Takagi, R.; Zhuang, Q. Convex resource theory of non-Gaussianity. Phys. Rev. A 2018, 97, 062337. [CrossRef]

5. Albarelli, F.; Genoni, M.G.; Paris, M.G.A.; Ferraro, A. Resource theory of quantum non-Gaussianity and Wigner negativity. Phys. Rev. A 2018, 98, 052350. [CrossRef]

6. Zhang, D.; Cai, Y.; Zheng, Z.; Barral, D.; Zhang, Y.; Xiao, M.; Bencheikh, K. Non-Gaussian nature and entanglement of spontaneous parametric nondegenerate triple-photon generation. Phys. Rev. A 2021, 103, 013704. [CrossRef]

7. Genoni, M.G.; Paris, M.G.A.; Banaszek, K. Measure of the non-Gaussian character of a quantum state. Phys. Rev. A 2007, 76, 042327. [CrossRef]

8. Genoni, M.G.; Paris, M.G.A.; Banaszek, K. Quantifying the non-Gaussian character of a quantum state by quantum relative entropy. Phys. Rev. A 2008, 78, 060303. [CrossRef]

9. Genoni, M.G.; Paris, M.G.A. Quantifying non-Gaussianity for quantum information. Phys. Rev. A 2010, 82, 052341. [CrossRef]

10. Barbieri, M.; Spagnolo, N.; Genoni, M.G.; Ferreyrol, F.; Blandino, R.; Paris, M.G.A.; Grangier, P.; Tualle-Brouri, R. Non-Gaussianity of quantum states: An experimental test on single-photon-added coherent states. Phys. Rev. A 2010, 82, 063833. [CrossRef]

11. Mandilara, A.; Karpov, E.; Cerf, N.J. Gaussianity bounds for quantum mixed states with a positive Wigner function. J. Phys. Conf. Ser. 2010, 254, 012011. [CrossRef]

12. Filip, R.; Mišta, L., Jr. Detecting quantum states with a positive Wigner function beyond mixtures of Gaussian states. Phys. Rev. Lett. 2011, 106, 200401. [CrossRef]

13. Ježek, M.; Straka, I.; Mičuda, M.; Dušek, M.; Fiurášek, J.; Filip, R. Experimental Test of the Quantum Non-Gaussian Character of a Heralded Single-Photon State. Phys. Rev. Lett. 2011, 107, 213602. [CrossRef] 
14. Xu, X.X.; Yuan, H.C.; Hu, L.-Y.; Fan, H.-Y. Non-Gaussianity of photon-added-then-subtracted squeezed vacuum state. Optik 2012, 123, 16-20. [CrossRef]

15. Ivan, J.S.; Kumar, M.S.; Simon, R. A measure of non-Gaussianity for quantum states. Quantum Inf. Process 2012, 11, 853-872. [CrossRef]

16. Mandilara, A.; Cerf, N.J. Quantum uncertainty relation saturated by the eigenstates of the harmonic oscillator. Phys. Rev. A 2012, 86, 030102. [CrossRef]

17. Ghiu, I.; Marian, P.; Marian, T.A. Measures of non-Gaussianity for one-mode field states. Phys. Scr. 2013, T153, 014028. [CrossRef]

18. Genoni, M.G.; Palma, M.L.; Tufarelli, T.; Olivares, S.; Kim, M.S.; Paris, M.G.A. Detecting quantum non-Gaussianity via the Wigner function. Phys. Rev. A 2013, 87, 062104. [CrossRef]

19. Marian, P.; Marian, T.A. Relative entropy is an exact measure of non-Gaussianity. Phys. Rev. A 2013, 88, 012322. [CrossRef]

20. Lachman, L.; Filip, R. Robustness of quantum nonclassicality and non-Gaussianity of single-photon states in attenuating channels. Phys. Rev. A 2013, 88, 063841. [CrossRef]

21. Mandilara, A.; Karpov, E.; Cerf, N.J. Purity- and Gaussianity-bounded uncertainty relations. J. Phys. A Math. Theor. 2014, 47, 045302. [CrossRef]

22. Hughes, C.; Genoni, M.G.; Tufarelli, T.; Paris, M.G.A.; Kim, M.S. Quantum non-Gaussianity witnesses in phase space. Phys. Rev. A 2014, 90, 013810. [CrossRef]

23. Xu, X.-X.; Luo, W.-W.; Zhang, H.-L.; Ma, S.-J. Nonclassical and non-Gaussian properties of states generated by the superposed photon added-and-subtracted operation on squeezed vacuum. Optik 2014, 125, 4190-4195. [CrossRef]

24. Seshadreesan, K.P.; Dowling, J.P.; Agarwal, G.S. Non-Gaussian entangled states and quantum teleportation of Schrödinger-cat states. Phys. Scr. 2015, 90, 074029. [CrossRef]

25. Park, J.; Zhang, J.; Lee, J.; Ji, S.-W.; Um, M.; Lv, D.; Kim, K.; Nha, H. Testing nonclassicality and non-Gaussianity in phase space. Phys. Rev. Lett. 2015, 114, 190402. [CrossRef]

26. Xiang, S.-H.; Song, K.-H. Quantum non-Gaussianity of single-mode Schrödinger cat states based on kurtosis. Eur. Phys. J. D 2015, 69, 260. [CrossRef]

27. Son, W. Role of quantum non-Gaussian distance in entropic uncertainty relations. Phys. Rev. A 2015, 92, 012114. [CrossRef]

28. Park, J.; Nha, H. Demonstrating nonclassicality and non-Gaussianity of single-mode fields: Bell-type tests using generalized phase-space distributions. Phys. Rev. A 2015, 92, 062134. [CrossRef]

29. Xiao, J.; Xiong, C.; Yuan, H.-C.; Chen, L.; Zhu, X.-F. Non-positive Wigner function and non-Gaussianty generated by multiple creation-then-annihilation coherent state. Int. J. Theor. Phys. 2016, 55, 1719-1727. [CrossRef]

30. Hertz, A.; Karpov, E.; Mandilara, A.; Cerf, N.J. Detection of non-Gaussian entangled states with an improved continuous-variable separability criterion. Phys. Rev. A 2016, 93, 032330. [CrossRef]

31. Park, J.; Lu, Y.; Lee, J.; Shen, Y.; Zhang, K.; Zhang, S.; Zubairy, M.S.; Kim, K.; Nha, H. Revealing nonclassicality beyond Gaussian states via a single marginal distribution. Proc. Natl. Acad. Sci. USA 2017, 114, 891-896. [CrossRef]

32. Park, J.; Lee, J.; Ji, S.-W.; Nha, H. Quantifying non-Gaussianity of quantum-state correlation. Phys. Rev. A 2017, 96, 052324. [CrossRef]

33. Happ, L.; Efremov, M.A.; Nha, H.; Schleich, W.P. Sufficient condition for a quantum state to be genuinely quantum non-Gaussian. New J. Phys. 2018, 20, 023046. [CrossRef]

34. Xiang, S.-H.; Wen, W.; Zhao, Y.-J.; Song, K.-H. Evaluation of the non-Gaussianity of two-mode entangled states over a bosonic memory channel via cumulant theory and quadrature detection. Phys. Rev. A 2018, 97, 042303. [CrossRef]

35. Kühn, B.; Vogel, W. Quantum non-Gaussianity and quantification of nonclassicality. Phys. Rev. A 2018, 97, 053823. [CrossRef]

36. Berrada, K.; Eleuch, H. Measure of non-Gaussianity for photon-added nonlinear coherent states. Int. J. Geom. Meth. Mod. Phys. 2018, 15, 1850158. [CrossRef]

37. Baek K.; Nha, H. Non-Gaussianity and entropy-bounded uncertainty relations: Application to detection of non-Gaussian entangled states. Phys. Rev. A 2018, 98, 042314. [CrossRef]

38. Xiang, S.-H.; Zhao, Y.-J.; Xiang, C.; Wen, W.; Long, X.-W. A method for efficiently estimating non-Gaussianity of continuousvariable quantum states. Eur. Phys. J. D 2020, 74, 16. [CrossRef]

39. Fu, S.; Luo, S.; Zhang, Y. Quantifying non-Gaussianity of bosonic fields via an uncertainty relation. Phys. Rev. A 2020, 101, 012125 . [CrossRef]

40. Zhang, Y.; Luo, S. Quantifying non-Gaussianity via the Hellinger distance. Theor. Math. Phys. 2020, 204, 1046-1058. [CrossRef]

41. Hloušek, J.; Ježek, M.; Fiurášek, J. Direct experimental certification of quantum non-Gaussian character and Wigner function negativity of single-photon detectors. Phys. Rev. Lett. 2021, 126, 043601. [CrossRef] [PubMed]

42. Hillery, M. Nonclassical distance in quantum optics. Phys. Rev. A 1987, 35, 725-732. [CrossRef] [PubMed]

43. Dodonov, V.V.; Man'ko, O.V.; Man'ko, V.I.; Wünsche, A. Energy-sensitive and "classical-like" distances between quantum states Phys. Scr. 1999, 59, 81-89. [CrossRef]

44. Dodonov, V.V.; Man'ko, O.V.; Man'ko, V.I.; Wünsche, A. Hilbert-Schmidt distance and nonclassicality of states in quantum optics. J. Mod. Opt. 2000, 47, 633-654. [CrossRef]

45. Zyczkowski, K.; Slomczynski, W. The Monge metric on the sphere and geometry of quantum states. J. Phys. A Math. Gen. 2001, 34, 6689-6722. [CrossRef]

46. Malbouisson, J.M.C.; Baseia, B. On the measure of nonclassicality of field states. Phys. Scr. 2003, 67, 93-98. [CrossRef] 
47. Dodonov, V.V.; Renó, M.B. Classicality and anticlassicality measures of pure and mixed quantum states. Phys. Lett. A 2003, 308, 249-255. [CrossRef]

48. Marian, P.; Marian, T.A.; Scutaru, H. Distinguishability and nonclassicality of one-mode Gaussian states. Phys. Rev. A 2004, 69, 022104. [CrossRef]

49. Klimov, A.B.; Sánchez-Soto, L.L.; Yustas, E.C.; Söderholm, J.; Björk, G. Distance-based degrees of polarization for a quantum field. Phys. Rev. A 2005, 72, 033813. [CrossRef]

50. Rivas, A.; Luis, A. Intrinsic metrological resolution as a distance measure and nonclassical light. Phys. Rev. A 2008, 77, 063813. [CrossRef]

51. Miranowicz, A.; Bartkiewicz, K.; Pathak, A.; Peřina, J., Jr.; Chen, Y.-N.; Nori, F. Statistical mixtures of states can be more quantum than their superpositions: Comparison of nonclassicality measures for single-qubit states. Phys. Rev. A 2015, $91,042309$. [CrossRef]

52. Marian, P.; Marian, T.A. A geometric measure of non-classicality. Phys. Scr. 2020, 95, 054005. [CrossRef]

53. Jozsa, R. Fidelity for mixed quantum states. J. Mod. Opt. 1994, 41, 2315-2323. [CrossRef]

54. Schrödinger, E. Zum Heisenbergschen Unschärfeprinzip. In Sitzungsberichte der Preussischen Akademie der Wissenschaften. Physikalisch-Mathematische Klasse; Akademie der Wissenschaften: Berlin, Germany, 1930; pp. 296-303.

55. Robertson, H.P. A general formulation of the uncertainty principle and its classical interpretation. Phys. Rev. 1930, $35,667$.

56. Dodonov, V.V.; Man'ko, V.I. Generalization of the Uncertainty Relations in Quantum Mechanics. In Invariants and the Evolution of Nonstationary Quantum Systems; Markov, M.A., Ed.; Proceedings of Lebedev Physics Institute; Nova Science: Commack, NY, USA, 1989; Volume 183, pp. 3-101.

57. Ivan, J.S.; Mukunda, N.; Simon, R. Moments of non-Gaussian Wigner distributions and a generalized uncertainty principle: I. The single-mode case. J. Phys. A Math. Gen. 2012, 45, 195305. [CrossRef]

58. Citeli, M.F.; Dantas, V.M.; Dodonov, V.V. Minimal products of coordinate and momentum uncertainties of high orders: Significant and weak high-order squeezing. Entropy 2020, 22, 980. [CrossRef]

59. Lynch, R.; Mavromatis, H.A. Nth (even)-order minimum uncertainty products. J. Math. Phys. 1990, 31, 1947-1951. [CrossRef]

60. Wigner, E.P.; Yanase, M.M. Information Contents of Distributions. Proc. Nat. Acad. Sci. USA 1963, 49, 910-918. [CrossRef]

61. Dodonov, V.V.; Malkin, I.A.; Man'ko, V.I. Even and odd coherent states and excitations of a singular oscillator. Physica 1974, 72, 597-615. [CrossRef]

62. Bužek, V.; Knight, P.L. Quantum interference, superposition states of light, and nonclassical effects. In Progress in Optics; Wolf, E., Ed.; North Holland: Amsterdam, The Netherlands, 1995; Volume 34, pp. 1-158.

63. Man'ko, V.I. Even and odd coherent states and tomographic representation of quantum mechanics and quantum optics. In Theory of Nonclassical States of Light; Dodonov, V.V.; Man'ko, V.I., Eds.; Taylor \& Francis: London, UK, 2003 ; pp. $219-240$.

64. Tang, X.-B.; Gao, F.; Wang, Y.-X.; Wu, J.-G.; Shuang, F. Non-Gaussian features from excited squeezed vacuum state. Opt. Commun. 2015, 345, 86-98. [CrossRef]

65. Sanders, B.C. Superposition of two squeezed vacuum states and interference effects. Phys. Rev. A 1989, 39, 4284-4287. [CrossRef]

66. Puri, R.R. Mathematical Methods of Quantum Optics; Springer: Berlin/Heidelberg, Germany, 2001.

67. Wünsche, A. Squeezed states. In Theory of Nonclassical States of Light; Dodonov, V.V., Man'ko, V.I., Eds.; Taylor \& Francis: London, UK, 2003; pp. 95-152.

68. Xin, Z.Z.; Wang, D.B.; Hirayama, M.; Matumoto, K. Even and odd two-photon coherent states of the radiation field. Phys. Rev. A 1994, 50, 2865-2869. [CrossRef]

69. Barbosa, Y.A.; Marques, G.C.; Baseia, B. Generalized superposition of two squeezed states: Generation and statistical properties. Physica A 2000, 280, 346-361. [CrossRef]

70. Hach, E.E., III; Gerry, C.C. Four photon coherent states. Properties and generation. J. Mod. Opt. 1992, 39, 2501-2517. [CrossRef]

71. Lynch, R. Simultaneous fourth-order squeezing of both quadrature components. Phys. Rev. A 1994, 49, 2800-2805. [CrossRef]

72. Souza Silva, A.L.; Mizrahi, S.S.; Dodonov, V.V. Effect of phase-sensitive reservoir on the decoherence of pair-cat coherent states. J. Russ. Laser Res. 2001, 22, 534-544. [CrossRef]

73. Zurek, W.H. Sub-Planck structure in phase space and its relevance for quantum decoherence. Nature 2001, 412, 712-717. [CrossRef]

74. Lee, S.-Y.; Lee, C.-W.; Nha, H.; Kaszlikowski, D. Quantum phase estimation using a multi-headed cat state. J. Opt. Soc. Am. B 2015, 32, 1186-1192. [CrossRef]

75. Mirrahimi, M.; Leghtas, Z.; Albert, V.V.; Touzard, S.; Schoelkopf, R.J.; Jiang, L.; Devoret, M.H. Dynamically protected cat-qubits: A new paradigm for universal quantum computation. New J. Phys. 2014, 16, 045014. [CrossRef]

76. Akhtar, N.; Sanders, B.C.; Navarrete-Benlloch, C. Sub-Planck structures: Analogies between the Heisenberg-Weyl and SU(2) groups. Phys. Rev. A 2021, 103, 053711. [CrossRef]

77. Prudnikov, A.P.; Brychkov, Y.A.; Marichev, O.I. Integrals and Series. Volume 2. Special Functions; Taylor \& Francis: London, UK, 2002.

78. Dodonov, V.V. Wigner functions and statistical moments of quantum states with definite parity. Phys. Lett. A 2007, 364, 368-371. [CrossRef]

79. Hudson, R.L. When is the Wigner quasi-probability density non-negative? Rep. Math. Phys. 1974, 6, 249-252. [CrossRef]

80. Szabo, S.; Adam, P.; Janszky, J.; Domokos, P. Construction of quantum states of the radiation field by discrete coherent-state superpositions. Phys. Rev. A 1996, 53, 2698-2710. [CrossRef] [PubMed]

81. Napoli, A.; Messina, A. Generalized even and odd coherent states of a single bosonic mode. Eur. Phys. J. D 1999, 5, 441-445. [CrossRef] 
82. José, W.D.; Mizrahi, S.S. Generation of circular states and Fock states in a trapped ion. J. Opt. B Quantum Semiclass. Opt. 2000, 2, 306-314. [CrossRef]

83. Su, D.; Myers, C.R.; Sabapathy, K.K. Conversion of Gaussian states to non-Gaussian states using photon-number-resolving detectors. Phys. Rev. A 2019, 100, 052301. [CrossRef]

84. Oh, C.; Lee, C.; Banchi, L.; Lee, S.-Y.; Rockstuhl, C.; Jeong, H. Optimal measurements for quantum fidelity between Gaussian states and its relevance to quantum metrology. Phys. Rev. A 2019, 100, 012323. [CrossRef] 